\title{
Did Credit Rating Agencies Make Unbiased Assumptions on CDOs?
}

\author{
By JOHN M. GRIFFIN AND DRAGON YONGJUN TANG*
}

Financial intermediaries are often in conflicting situations where large, short-term profits can be made by deviating from conventional standards. The frequency and severity of such deviations is a source of substantial disagreement. During the 'dot-com’ period, equity analysts knowingly inflated their ratings on internet stocks that their banks underwrote and most large investment banks engaged in questionable IPO allocation practices (John M. Griffin, Jeffrey H. Harris, and Selim Topaloglu (2007)). However, other studies suggest that such behaviors are exceptions rather than the norm. For example, Hamid Mehran and Rene M. Stulz (2007, page 293) summarize that "the academic literature on conflicts of interest, using large samples, reaches conclusions that are weaker and often more benign than the conclusions drawn by journalists and politicians.” The credit crisis provides a new testing ground for such debate.

Collateralized Debt Obligations (CDOs), a pool of debt securities sold to investors in prioritized tranches, are at the heart of the credit crisis of 2007-2009. The stellar growth of the CDO market before the crisis and its sudden collapse has stimulated vigorous discussion on agency conflicts. In particular, rating agencies are accused of having made unrealistic assumptions on structured finance products to issue inflated AAA ratings. Rating agencies admit that their correlation assumptions were too low, but maintain that the assumptions were extrapolated from historical data and not biased by conflicting incentives. We analyze potential rating bias through a straightforward approach-we compare the assumptions used in the same CDO valuation model performed by two divisions within the same rating agency with different financial incentives. The ratings division interacts with clients and assigns CDO ratings. Their job is both to bring in business as well as adhere to high standards. A common concern in such a 
business model is that the business side might be overly aggressive in their assumptions in order to gain market share. This might be particularly true in deals such as CDOs where complexity makes it difficult for others to easily verify rating quality in the short run. In contrast, the surveillance division monitors the performance of the CDO.

Our results indicate that the surveillance team calculations were more accurate than those of the ratings team and also more economically meaningful for future performance. However, downgrading signals from surveillance results seem to be ignored. As we will discuss, our findings are consistent with the rating agency supporting inflated CDO ratings and inconsistent with other explanations regarding CDO completeness, timing, or collateral deterioration in 2007.

Our paper adds to a growing literature on lapses in structured finance credit ratings. Adam Ashcraft, Paul Goldsmith-Pinkham, and James Vickery (2010) show that rating agencies failed to incorporate simple information into mortgage-backed securities ratings. Joshua D. Coval, Jakub W. Jurek, and Erik Stafford (2009a, and b) argue that CDO pricing should incorporate catastrophe risk and parameter uncertainty. John M. Griffin and Dragon Tang (2011) find that in granting AAA ratings a credit rating agency made large inflationary adjustments beyond their standard model. ${ }^{1}$

\section{Rating Assumption Changes from New Issue to First Surveillance}

\section{A. Brief Institutional Background}

Determining the credit quality of individual assets in the collateral pool and the default correlation between the assets that feed into the quantitative CDO evaluation model are two of the primary tasks of the ratings analysts. Assets currently rated by the rating agency are counted at face value while assets rated by a different rating agency are typically notched down. A rating

\footnotetext{
${ }^{1}$ Patrick Bolton, Xavier Freixas, and Joel Shapiro (2009), Vasiliki Skreta and Laura Veldkamp (2009), and Francesco Sangiorgi and Chester S. Spatt (2010) examine credit rating shopping from a theoretical perspective.
} 
analyst should analyze the credit quality of unrated assets. Rating agencies categorize collateral assets by sector and then use defined values for within- and across-type correlations. However, credit risk models do allow for these correlation assumptions to be customized. Adding to the challenge is the fact that the portfolio pool is often incomplete or partially ramped when CDO ratings are assigned. The ratings group must also foster the business relationships brought in by the sales team and interact with the investment bank underwriting the CDOs. A "Pre-Sale" and/or "New Issue” report is typically prepared by a ratings analyst and approved by a ratings committee around the time the rating is issued to facilitate the closing of the CDO. ${ }^{2}$

Rating agencies also promise continuous active surveillance after the initial credit rating on the CDO is assigned. The last section of S\&P's new issue and pre-sale reports discloses their surveillance policy: "The purpose of surveillance is to assess whether the rated notes are performing within the initial parameters and assumptions." The surveillance analyst receives collateral information from trustees and monitors CDO performance. If surveillance reports indicate that current ratings are no longer appropriate, a rating review will be conducted and the CDO notes could be upgraded or downgraded. The first surveillance report generally arrives after the rating is initially assigned.

Our focus is on the correlation and credit quality assumptions which are the key inputs of the CDO rating model. Both departments use the same ratings model. We call these inputs 'assumptions', but they are quantitative in the sense that rating agencies have a set of standard procedures to assign these values. Hence they are summary measures of the correlation and collateral quality, but judgment could play a role in the calculation process. From what is written in the press and from our discussions with industry insiders, we expect the ratings committee to

\footnotetext{
${ }^{2}$ Other details of the rating and modeling process can be found in Coval, Jurek, and Stafford (2009a and b), Efraim Benmelech and Jennifer Dlugosz (2009), and Griffin and Tang (2011).
} 
have more discretion than the surveillance group — the surveillance group is more reminiscent of a compliance or risk management division. While a surveillance department may be forced to corroborate the ratings department, they should have some autonomy and may not fully communicate with the ratings group.

\section{B. Data and Basic Statistics}

We obtain data from one of the three leading credit rating agencies, including two sets of reported CDO assumptions and outputs. One is machine- and hand-collected from pre-sale and new issue reports when the CDO was issued to investors at the closing time. The other information is collected from an online credit rating agency database containing the first surveillance reports when the CDO is fully operating. (Part of the data is used by Griffin and Tang (2011).) The intersection of the two data sources leaves 595 CDOs with both rating assumptions available. However, to focus on information that is timely, we restrict the dataset to the 355 CDOs with surveillance reports dated within 180 days of the initial rating assignment. Results for the full sample are similar and shown in the Online Appendix. The correlation measure reported by the rating agency is the ratio between the standard deviation of the CDO pool under the assumed correlation structure relative to the standard deviation of a CDO pool with completely uncorrelated assets. Aggregate portfolio risk is represented by the simulation output known as the scenario default rate (SDR). The AAA SDR is the portfolio loss expected to occur with a probability equal to the historical default frequency of AAA-rated corporate bonds.

The changes in correlation measure and average collateral rating between the first surveillance reports and the initial rating reports are plotted in Panel A of Figure 1. The figure shows that more correlation measure changes are positive (58.6 percent) as compared to negative (38.9 percent), indicating that the surveillance group estimates a higher default correlation than 
that used by the ratings group. Both the mean and median differences are highly statistically significant as reported in Table 1. On average, the correlation measure increases from rating assignment to the first surveillance report by a statistically significant 0.116 , which implies an economically large 14.9 percent increase in the correlation level. ${ }^{3}$

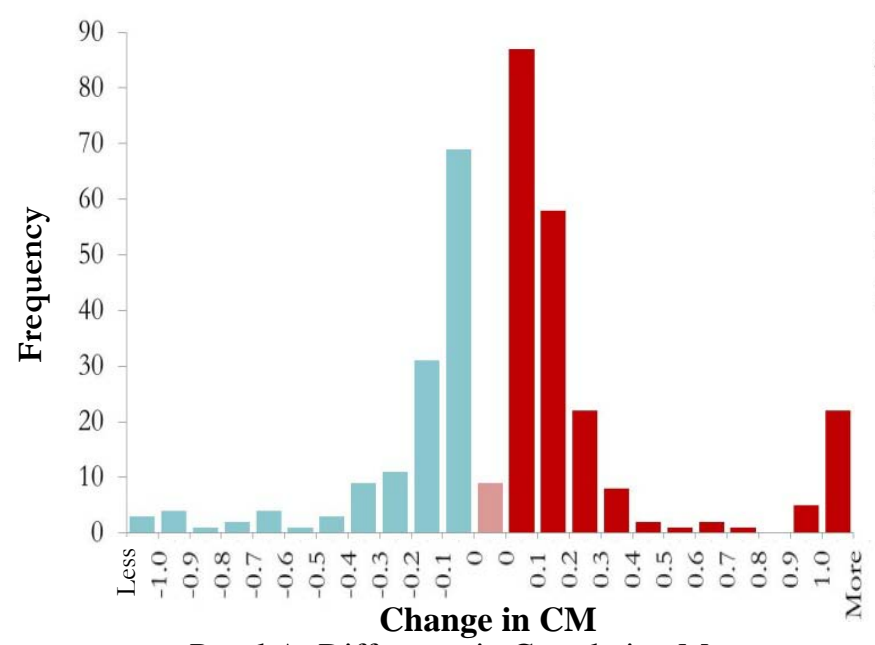

Panel A: Difference in Correlation Measure from Surveillance to Issuing Reports

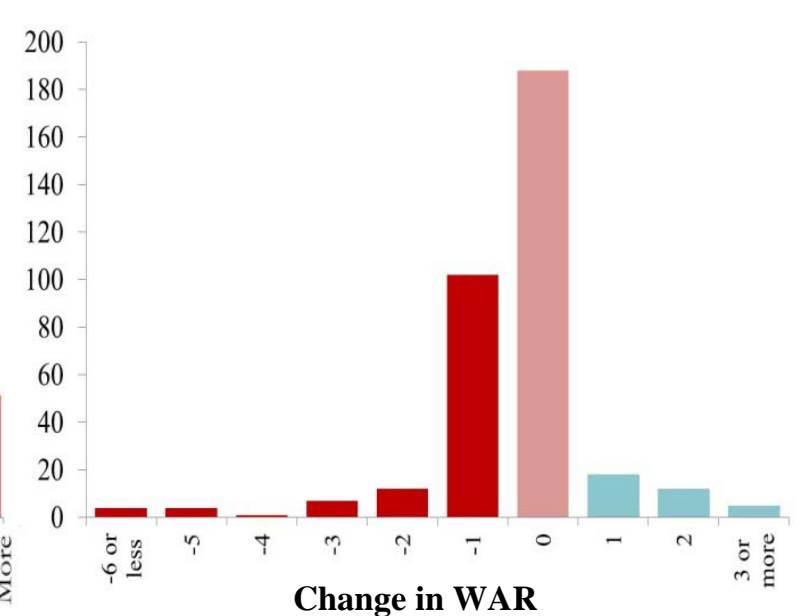

Panel B: Difference in Weighted Average Rating from Surveillance to Issuing Reports

Figure 1. Changes in Collateral Assumptions from Rating Assignment to First Surveillance. Notes: Illustrated are histograms for changes in collateral assumptions from rating assignment reports to first surveillance reports. The left panel illustrates changes in the default correlation measure (CM) assumption. The right panel illustrates changes in the weighted average rating (WAR) assumption. CM changes are in difference. WAR changes are in number of notches. The reporting gap is within 180 days. The sample covers 355 CDOs issued between 2002 and 2007.

Table 1 shows that the surveillance group calculates much more pessimistic collateral credit quality than that assumed by the ratings team. The surveillance group calculates collateral ratings that are one or more notches worse than that estimated by the ratings team for 36.8 percent of CDOs and collateral ratings one or more notches better in only 9.9 percent of CDOs, as shown by Panel B of Figure 1. On average, the surveillance group's collateral rating is lower by a statistically significant one-third of a notch.

\footnotetext{
${ }^{3}$ The average issuing report correlation measure is 1.78 . For the percentage calculation we subtract one since an asset with zero correlation will have a correlation measure of one.
} 


\section{Table 1}

Changes in Assumptions and Outputs from Rating Assignment to First Surveillance Report

\begin{tabular}{lccccccc}
\hline \multicolumn{7}{c}{ Panel A } \\
\hline$\Delta$ Correlation Measure & $\mathrm{N}$ & Mean & t-stat & Median & \% Positive & \% Negative & p-val \\
\hline Weighted Average Rating & 355 & 0.116 & $(2.74)$ & 0.04 & $58.6 \%$ & $38.9 \%$ & 0.0002 \\
& 353 & -0.377 & $(-4.79)$ & 0.00 & $9.9 \%$ & $36.8 \%$ & 0.0000 \\
\hline$\Delta$ Scenario Default Rate & 298 & 0.016 & Panel B & & & \\
\hline
\end{tabular}

Notes: This table presents summary statistics for the changes in the collateral assumptions and outputs from rating assignment reports to first surveillance reports. The reporting time gap is within 180 days. Sample CDOs are issued between 2002 and 2007. The first row in Panel A reports changes in the default correlation measure assumption. The second row reports changes in the weighted average rating assumption in number of notches. Panel B reports changes in scenario default rate (SDR). CM changes are in difference. Scenario Default Rate changes are in raw values. Column 'p-val' tests the likelihood of the positive/negative split relative to a null of $\mathrm{p}=.5$.

\section{Are the Correlation and Collateral Quality Changes Structural?}

If changes in the correlation and collateral quality assumptions are offset by changes elsewhere in the CDO structure (such as maturity), these changes would not affect the risk of the CDO. Changes in collateral assumptions feed directly into the assessment of portfolio risk, such as the scenario default rate. Panel B of Table 1 reports the AAA SDR. For the sample with SDRs we find that the first surveillance report SDRs are 1.6 percent higher than those in the initial ratings reports. The average SDR in the ratings reports is 32.5 percent. The 1.6 percent increase in SDR represents a five percent increase in portfolio risk assessed by the surveillance analysts. In Online Appendix Table OA.4 regressions indicate that an increase in correlation measure and deterioration in average collateral quality are indeed strongly related to the SDR increase. Hence, it does not seem that the correlation increases and average collateral quality changes are made up 
for elsewhere in the CDO structure. Additionally, this analysis indicates that these changes in assumptions lead to more risky CDOs than released to investors at the time of the initial rating.

We consider several possible explanations for the changes in the correlation measure and collateral quality. First, one straightforward potential reason for observing higher correlation and lower credit quality in surveillance than issuance reports is that the collateral pool changed between reports. Collateral composition change is more likely when the collateral pool is less ramped up at the issuing stage. Panel A of Table OA.2 shows that even for near fully and fully ramped CDOs, the changes in correlation and collateral quality are still significant. Surprisingly, the group with the lowest ramp-up fractions has smaller changes, although the sample size is much smaller. Second, collateral composition is more likely to change if the time between issuance and surveillance is longer. In Panel B of Table OA.2, we find that collateral quality deterioration is larger for longer time gaps, but the change in correlation is similar for the time gap of 0-3 months and 3-6 months. Third, the information environment could have changed from issuance to surveillance because of the mortgage market deterioration in 2007. We separately report the changes in 2007 and pre-crisis in Panel C of Table OA.2 and find that differences in correlations and especially collateral quality are large prior to 2007. We report changes in the correlation measure and weighted average rating over time in Figure OA.7 and find that the changes in correlation were about zero initially but became increasingly positive from 2005 to 2007.

Additionally, it is interesting to relate the findings to the type of deal. Sangiorgi and Spatt (2010) show that rating bias would only arise in an opaque environment for CDO ratings. ${ }^{4}$ Panel

\footnotetext{
${ }^{4}$ ABS CDOs and CDOs of CDOs are arguably more complex than plain vanilla CDOs based on bonds and loans (CBOs and CLOs). However, the underlying collateral for ABS CDOs and $\mathrm{CDO}^{2} \mathrm{~s}$ has often been previously rated,
} 
D of Table OA.2 shows that the correlation increase is most prevalent in ABS CDOs. Collateral quality differences are negative and similar for ABS CDOs, CLOs, and $\mathrm{CDO}^{2}$ s. Thus, while complexity for CDOs in general could have a role in the difference between the two groups, it is not clear that differential complexity within CDOs plays a role.

Hence, the changes in collateral quality and correlation assumptions are materially important but not explained by collateral composition changes, time between reports, or rapid changes in market conditions.

\section{Implications of Assumption Changes}

It is unclear whether the assumption changes between reports are economically important. The future performance of CDOs will detail which group, issuance or surveillance, is more accurate, and whether CDO investors are materially affected by those systematic changes in assumptions. Following Griffin and Tang (2011), we collect the rating changes for originally AAA-rated CDO tranches. ${ }^{5}$

Table 2 reports the ordered logistic regression results with the number of notches downgraded from AAA as the dependent variable. The change in assumed correlation significantly positively predicts future downgrading. This indicates that the surveillance team was more correct than the ratings analyst team. The odds ratio of 2.05 indicates that the odds of being downgraded is 2.05 times greater when the rating analysts' under-estimation is one unit below the surveillance analyst. The specifications are also robust to controls for CDO type and

while CLOs seem likely to contain a higher proportion of unrated underlying loans that require more subjective evaluation of collaterals.

${ }^{5}$ For CDOs with multiple AAA-rated tranches, we count the worst rating downgrading. The AAA downgrade ranges from 0 if the AAA rating is maintained throughout the life of the CDO to 21 if the tranche has defaulted. 
vintage. The change in the SDR is similarly significant. ${ }^{6}$ These findings are quite robust as shown with hazard models, ordered probit, plain probit, and OLS (Tables OA.8 and OA.9).

Table 2

\section{Rating Assumption Changes Predicting AAA Downgrading}

\begin{tabular}{lcc}
\hline & $(1)$ & $(2)$ \\
\hline$\Delta$ Correlation Measure & 2.05 & \\
& $(3.77)$ & \\
$\Delta$ Weighted Average Rating & 0.99 & \\
$\Delta$ Weighted Average Maturity & $(-0.13)$ & \\
& 1.14 & \\
Change in SDR & $(1.44)$ & \\
& & 830.09 \\
ABS CDO & 19.19 & $(3.33)$ \\
& $(9.54)$ & $(8.59)$ \\
CDO $^{2}$ & 3.81 & 5.82 \\
& $(1.33)$ & $(1.77)$ \\
Year 2004 & 0.44 & 0.31 \\
& $(-0.97)$ & $(-1.21)$ \\
Year 2005 & 1.71 & 1.71 \\
& $(0.82)$ & $(0.69)$ \\
Year 2006 & 2.94 & 2.86 \\
& $(1.69)$ & $(1.38)$ \\
Year 2007 & 3.20 & 3.19 \\
& $(1.73)$ & $(1.48)$ \\
\hline Number of Obs. & 347 & 294 \\
Pseudo R & 0.144 & 0.147 \\
\hline
\end{tabular}

Notes: This table reports ordered logistic regression results. The dependent variable is the number of notches downgraded from the initial AAA ratings. Independent variables are changes, from rating assignment to first surveillance, in the default correlation measure (CM) assumption, the weighted average rating (WAR) assumption, the weighted average maturity (WAM) assumption, and scenario default rate (SDR). Reported are odds ratios and z-statistics in parenthesis.

Assumption changes would be irrelevant if investors do not rely on rating agency assumptions or fully anticipate surveillance changes. We find that the market spreads at issuance did not seem to reflect the future information that the SDR or the correlation and collateral quality assumptions would deteriorate (in Table OA.10).

\footnotetext{
${ }^{6}$ Because the change in the correlation measure is so strongly related to the change in SDR, one faces problems with colinearity when including both variables, but we find that the change in the correlation measure prevails.
} 
Why is surveillance analysis more accurate? It may simply be that surveillance analysts have more resources or are more talented. However, common perception is that ratings analysts received higher compensation and more staffing than surveillance teams. Surveillance analysts are less influenced by conflicts of interest, and hence could make more objective assumptions.

If the rating agency had new information from the surveillance group and acted on it then it would indicate that the rating agency was learning from the surveillance team and trying to correct mistakes made by the ratings group. However, if the ratings agency did not act on information coming from the surveillance group, then this would indicate that the firm was compromising its standards. Since the AAA SDR increases for some deals, we can assess whether an increase in the SDR would have mattered for the rating agencies' key rating criteria.

We examine if the break-even default rate (BDR) from the cash flow model is greater than the SDR as discussed by Griffin and Tang (2011). Although our surveillance data does not contain a BDR, we evaluate the surveillance team SDR relative to the BDR in the issuing reports. If the BDR decreases for a deteriorating CDO (a natural case), our estimation for BDRs from issuing reports will be too high and lead to fewer rejections than if we had surveillance BDRs. Nevertheless, we still find that 19.7 percent of CDOs have at least one AAA tranche (and 20.1 percent of tranches) that fails to pass the test for granting an AAA rating. We verify that those CDO tranches were not downgraded before the first surveillance date. Hence, it seems that these CDO tranches would not have warranted the 'AAA' rating. If rating agencies did indeed ignore such important surveillance information, it provides strong evidence that the firm was going beyond their stated standards.

\section{Summary and Discussion}


We find that assigned CDO ratings at issuance by the ratings group are based on more aggressive assumptions than the surveillance calculations after issuance. This difference does not appear to be explained by changes in collateral composition, the length of time between reports, or the collapse of the subprime mortgage market. Changes in collateral assumptions by the surveillance group predict future downgrading. Hence, the surveillance reports, although they appear shortly after issuance, are more accurate than the rating issuance reports.

Consistent with the conflicts of interest hypothesis, the assumptions were more favorable in the group which brought in the business and interacted directly with the investment banks. Also consistent with trying to maintain high ratings, the rating agency did not seemingly act on downgrading signals from the surveillance department. Since the breakdown in CDO credit ratings was at the heart of the credit crisis of 2007-2009, our findings suggest that conflicts of interest may be much more economically important than previously surmised.

\section{References}

Ashcraft, Adam B., Paul Goldsmith-Pinkham, and James I. Vickery. 2010. "MBS Ratings and the Mortgage Credit Boom.” FRB of New York Staff Report No. 449.

Benmelech, Efraim, and Jennifer Dlugosz. 2009. "The Alchemy of CDO Credit Ratings.” Journal of Monetary Economics, 56(5): 617-634.

Bolton, Patrick, Xavier Freixas, and Joel D. Shapiro. 2009. “The Credit Ratings Game.” NBER Working paper No. 14712.

Coval, Joshua D., Jakub W. Jurek, and Erik Stafford. 2009a. "Economic catastrophe bonds.” American Economic Review 99(3): 628-666.

Coval, Joshua D., Jakub W. Jurek, and Erik Stafford. 2009b. "The Economics of Structured Finance.” Journal of Economic Perspectives, 23(1): 3-25. 
Griffin, John M., Jeffrey H. Harris, and Selim Topaloglu. 2007. "Why are IPO Investors Net Buyers through Lead Underwriters?” Journal of Financial Economics, 85(2): 518-551.

Griffin, John M., and Dragon Yongjun Tang. 2011. “Did Subjectivity Play a Role in CDO Credit Ratings?” University of Texas Working Paper.

Mehran, Hamid, and Rene M. Stulz. 2007. "The Economics of Conflicts of Interest in Financial Institutions.” Journal of Financial Economics, 85(2): 267-296.

Sangiorgi, Francesco, and Chester Spatt. 2010. "Equilibrium credit ratings and policy.” Carnegie Mellon University Working Paper.

Skreta, Vasiliki, and Laura Veldkamp. 2009. "Rating Shopping and Asset Complexity: A Theory of Rating Inflation.” Journal of Monetary Economics, 56(5): 678-695. 